\title{
The utility of basic blood counts, WBC histogram and $\mathrm{C}$-reactive protein in detecting malaria
}

\author{
Jun Nishimura ${ }^{1 *}$, Parag Dharap ${ }^{2}$ and Sebastien Raimbault ${ }^{3}$
}

\begin{abstract}
Background: Hematology analyzers display abnormal parameters during malaria infection providing insightful information for suspecting and assessing malaria infection. The goal of this study is to demonstrate the potential of a three-part differential hematology analyzer to assess malaria, provide information about the parasitemia, and discuss the importance of combining C-reactive protein (CRP) with hematology parameters to obtain further information about the malaria infection.

Methods: The present study shows the results of a case-control study during the monsoon season of years 2018 and 2019 in Mumbai, India. The study considers 1008 non-malaria febrile cases, 209 P. vivax and 31 P. falciparum positive malaria samples, five cases of mixed P. vivax and P. falciparum infection, and three co-infection cases of $P$. vivax and dengue. Raw data from the three-part analyzer LC-667G CRP (HORIBA) and the corresponding microscopic findings (golden standard for diagnosis of malaria) were obtained for each sample.

Results: The medians of platelet counts (PLT) were 102.5, 109.0, and $223.0 \times 10^{3} / \mu \mathrm{L}$, while CRP medians were 67.4, 81.4 and $10.4 \mathrm{mg} / \mathrm{L}$ in P. vivax, P. falciparum and control groups respectively $(\mathrm{p}<0.001$ in Mann-Whitney $U$ tests between malaria and control groups). Compared with negative samples, platelets counting less than $161.5 \times 10^{3} / \mu \mathrm{L}$ were observed on malaria patients (OR 19.12,95\% Cl 11.89-30.75). Especially in P. vivax cases, an abnormal peak was frequently observed in the white blood cells (WBC) histogram around the $37 f \mathrm{~L}$ channel. The events counted around that channel showed a linear correlation with the counting of red blood cells infected predominantly with larger parasitic forms. Parameters like CRP ( $r s=0.325, p<0.001)$, WBC ( $r s=0.285, p<0.001)$ and PLT ( $r s=-0.303, p<0.001)$ were correlated with the parasitemia of $P$. vivax samples. Between the malaria and dengue groups, the highest area under the receiver operating characteristic curve was observed on CRP $(0.867, C R P \geq 26.85 \mathrm{mg} / \mathrm{L})$.
\end{abstract}

Conclusions: A three-part differential hematology analyzer has the potential to not only trigger malaria diagnosis confirmation but also assess the severity of the infection when CRP is considered.

Keywords: Malaria infection, Hematology analyzer, WBC histogram, CRP

${ }^{*}$ Correspondence: jun.nishimura@horiba.com

${ }^{1}$ Horiba Medical, Kyoto, Japan

Full list of author information is available at the end of the article

\begin{abstract}
Background
According to the $\mathrm{WHO}$ an estimated 228 million cases of malaria occurred in 2018 worldwide and $85 \%$ of this burden was located in 19 sub-Saharan Africa countries and India. For rapid disease management and surveillance of malaria, the WHO recommends two main strategies to eradicate malaria: closing the gaps of healthcare access
\end{abstract}

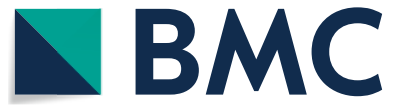

(c) The Author(s) 2021. Open Access This article is licensed under a Creative Commons Attribution 4.0 International License, which permits use, sharing, adaptation, distribution and reproduction in any medium or format, as long as you give appropriate credit to the original author(s) and the source, provide a link to the Creative Commons licence, and indicate if changes were made. The images or other third party material in this article are included in the article's Creative Commons licence, unless indicated otherwise in a credit line to the material. If material is not included in the article's Creative Commons licence and your intended use is not permitted by statutory regulation or exceeds the permitted use, you will need to obtain permission directly from the copyright holder. To view a copy of this licence, visit http://creativecommons.org/licenses/by/4.0/. The Creative Commons Public Domain Dedication waiver (http://creativeco mmons.org/publicdomain/zero/1.0/) applies to the data made available in this article, unless otherwise stated in a credit line to the data. 
between urban and rural areas, and diagnosis (before antimalarial treatment) by microscopy or malaria rapid diagnostic tests (RDTs) in all patients with malaria suspicion. Fever is the main manifestation for suspicion of malaria infection. In malaria endemic areas, it acts as a trigger for parasitology testing [1].

RDTs are currently replacing microscopy, the golden standard for diagnosing malaria, especially in endemic countries. Thus, complementary information should be considered to ensure correct interpretation and correct disease management particularly in surveillance programs [2]. The two main limitations of RDTs are the impossibility to obtain parasitemia quantification and, particularly in antibody based RDTs, the persistent positivity after treatment [2]. Microscopy can overcome both limitations by quantifying the parasite density, however the process is highly time-consuming.

\section{Malaria detection in standard automated cell counters (ACCs)}

The potential to diagnose clinically unsuspected cases of malaria for patients subjected to complete blood count $(\mathrm{CBC})$ is one of the main benefits of flagging malaria in hematology analyzers [3]. A comprehensive summary of studies for diagnosing malaria by ACCs without a specific malaria-detection design is well described by Campuzano-Zuluaga [3], in which many of the referred studies provide performances to detect malaria related to abnormalities in the white blood cell (WBC) differentiation channel. ACCs with cytometry capable of differentiating five WBC populations show a clear clustering for malaria population due to specific scattering of infected red blood cells (iRBCs) that are resistant to lyse and/or produce scatters due to residuals of the parasite infection. Pseudoeosinophilia resulting from neutrophils containing haemozoin has been reported as an abnormality caused by malaria infection [4-6]. Fourcade et al. demonstrated that large activated monocytes are present in malaria infections by measuring the standard deviation of the volume of lymphocytes (LYM) and monocytes (MON), calculating a malaria factor with sensitivity of $96.9 \%$ and specificity of $82.5 \%$ [7]. Many studies have identified a particular cluster by inspecting different angles of light after moderate lysing of red blood cells (RBC) without using extra reagents. However the majority of these studies have a lower performance to detect small or immature forms of parasites due to their low lysing resistance and small size. For example, a recent evaluation on a malaria parameter to evaluate the percentage of iRBCs in the BC-6800 (Mindray) showed a lower sensitivity to detect $P$. falciparum in comparison with detecting P. vivax
( $24.1 \%$ and $88.3 \%$, respectively). This is most probably due to the difficulty of detecting early stage parasites and their low nucleic acid content [8], which are prevalent in $P$. falciparum infections where trophozoites and schizonts are barely seen in peripheral blood.

In spite of all promising results from ACCs to identify malaria, fewer studies have been conducted for threepart differential ACCs. One study combined hemoglobin (HGB), total lymphocyte count (TLC), platelets (PLTs) and red cell distribution width (RDW) data in a KX-21 (Sysmex) achieving a $26.1 \%$ sensitivity and 96.3\% specificity in a study mainly consisting of $P$. vivax infected samples [9]. The majority of small ACCs utilize the Coulter-principle to classify WBCs in populations based on size and obtain an RBC distribution using limited reagents. Identifying intracellular presence of malaria parasites based only on abnormal WBC histogram distributions caused by the infection, poses a difficult challenge.

\section{C-reactive protein (CRP) to distinguish malaria}

CRP is a member of the pentraxin family of proteins, an acute-phase reactant and a biomarker for inflammation. Although CRP is not a specific biomarker for detecting malaria infection, several studies have identified CRP to be a potential marker to assess the severity of malaria infections [10-13]. A study in the south-western part of the Brazilian Amazon has shown evidence in the association of $P$. vivax malaria infection with inflammatory activation, including CRP, and cytokine imbalance [14].

Dengue and malaria infections, which frequently coexist in endemic areas, present with similar symptomatology and produce similar abnormalities in CBC parameters. CRP has been observed to be a useful biomarker to discriminate between malaria and dengue (Epelboin et al.) using CRP $>5 \mathrm{mg} / \mathrm{L}$ as a cut off to differentiate malaria from dengue, obtaining sensitivity of $99 \%$ and specificity of 35\% [15]. In addition, effective management and surveillance of malaria require a screening method facilitating quick transfer of patients to receive diagnostic confirmation and proper treatment. Point-of-care-testing (POCT) hematology analyzers providing information about malaria and other coexisting infections such as dengue could play a major role to support this line of action.

The goal of this study is to demonstrate the potential of a three-part differential hematology analyzer to assess malaria, provide information about the parasitemia, and discuss the importance of combining CRP with hematology parameters to obtain further information about the malaria infection. 


\section{Methods}

The present study shows the results of a case-control study involving comparison of raw instrument data from a three-part differential hematology analyzer with CRP measurement (LC-667G CRP, HORIBA) and corresponding microscopic findings of samples obtained from patients visiting Dr. Dharap's Diagnostic Centre, Mumbai, India during the monsoon season of years 2018 and 2019.

During monsoon season, the laboratory routinely caters to patients for diagnostic testing in cases of acute febrile illness which comprises of a CBC + CRP, peripheral examination of blood smear for malarial parasites and RDTs for malaria and dengue NS1, being an endemic area with coexistent infections. All groups considered for the current study were evaluated as patients with acute febrile illness. Documented historical data of known malaria cases, as well as dengue positive and negative cases were used for the study.

As being the golden standard for diagnosis, malaria positivity was determined solely by the examination of blood smears. We found four discordant cases between the blood smear inspection and the RDTs: one case was confirmed by blood smear but negative in both species RDTs, two samples were positive with $P$. vivax RDTs but negative in blood smear, and one sample was positive with $P$. falciparum RDT but negative in blood smear. For the current study, the historical documented data was further anonymized in coded fashion in order to protect patient confidentiality in accordance with local ethical or IRB guidelines, according to national and international standards for the conduct of clinical studies including 21 CFR Parts 50 and 56 and International Conference on Harmonization (ICH) E6-Good Clinical Practice Consolidated Guideline. Ethical clearance was not obtained as the study was carried as a retrospective review of the available raw instrument and historical patient data, without any collection of extra specimen or monetary charges to patients and did not involve any communication of results of the study to the clinician so as to affect the diagnostic and therapeutic management of the patients.

\section{Laboratory techniques}

Testing for 'Acute Febrile Illness' in the laboratory comprises of a full blood count, CRP value, peripheral examination of blood smear for malarial parasites, and RDTs for malaria and dengue NS1.

A full blood count is performed using a three-part differential hematology analyzer (LC-667G CRP, HORIBA) which additionally provides CRP level estimations. For peripheral blood smear examination the following technique is routinely used. Anticoagulated (K2-EDTA) blood of the patient is used to prepare thick \& thin blood smears for peripheral blood smear examination (PBSE). Both types of smear preparations are air dried. The thin preparation is fixed with methanol, whereas the thick blood smear is subjected to 'process of de-hemoglobinisation' with deionized water. Both smears are then stained by 'Field staining technique' using an automated slide stainer. Thin smears are examined for presence of malarial parasites and species identification, presence of platelet aggregates, macroplatelets and nucleated red blood cells, if any.

Malaria cases are further assessed for parasite density by counting parasite life cycle forms against 100 white blood cells by an experienced pathologist. The life cycle forms are grouped as small forms being ring and early ameboids, and large forms including late ameboids with ample parasitic material, schizonts and gametocytes.

For additional screening and confirmation of malaria cases, Rapid Malaria Antigen detection test kits manufactured by SD Biosensor Healthcare Pvt. Ltd., Gurugram, India using monoclonal anti-P. falciparum HRP-II $(0.75 \pm 0.15 \mu \mathrm{g})$ with monoclonal anti-P. vivax $\mathrm{pLDH}$ $(0.75 \pm 0.15 \mu \mathrm{g})$ respectively to detect presence of $P$. falc $i$ parum \& $P$. vivax related antigens are used.

For screening and diagnosis of dengue, RecombiLISA NS1 Antigen tests are performed on separate serum samples, utilizing pairs of specific polyclonal \& monoclonal anti-dengue antibodies of all four serotypes (DEN1, 2, 3, 4) and analytical sensitivity of $0.3 \mathrm{ng} / \mathrm{mL}$ for type $2 \mathrm{NS} 1$ antigen, manufactured by CTK Biotech, Inc., United States of America.

\section{Statistical analysis}

To determine the significance of the differences between the hematology parameters extracted from LC-667G CRP of positive \& negative samples of $P$. vivax \& P. falciparum samples respectively, as well as malaria \& dengue positive samples, Mann-Whitney U and Krustal-Wallis tests were applied due to their non-parametric distribution.

The Spearman's rank correlation coefficients were calculated for understanding the independent association of the inspected parameters with the parasites load of $P$. vivax positive samples and to understand if CRP is correlated with the age of patients in a group stratification of malaria species and patient sex. Moreover, to evaluate the hypothesis of CRP values being different between $P$. vivax and $P$. falciparum groups, Mann-Whitney $\mathrm{U}$ test was used to evaluate the difference in medians.

A receiver operating characteristic (ROC) curve, the area under the curve (AUC), sensitivity, specificity, and the odds ratios were calculated for evaluating the clinical significance to detect malaria positivity among 
fever patients and to differentiate malaria from dengue samples.

Due to the confounding factors in all the parameters from the analyzer, particularly CRP, and the limited distribution of samples, a matching method for reducing the risk of selection bias caused by the age and sex of patients was performed only in individual parameter comparisons (i.e., comparison of CRP boxplots between groups, CRP association with clinical variables and the receiver operating characteristic curve). Please refer to the Additional file 1: "Annex 1 QUADAS-2 analysis" for further information about the risk of bias for the present study.

All the whisker-plots and statistical analyses were computed with OriginPro 20219.8 .0200 and Excel 2016. The histogram graphs were created using $\mathrm{R}$ software 3.5.1.

\section{Results}

From the monsoon seasons of 2018 and 2019, based on selection criteria and availability of corresponding data, 209 (144 males, 65 females) P. vivax positive cases, 31 (22 males, 9 females) P. falciparum samples, five cases of mixed $P$. vivax and $P$. falciparum malaria infection (4 males, 1 female), and three co-infections of $P$. vivax and dengue ( 2 males, 1 female) were considered for the present study. Nonetheless, the mixed malarial infections, and the three $P$. vivax and dengue co-infection samples were excluded from all statistical tests. In addition, 1008 non-malaria febrile cases were examined including 197 (124 males, 73 females) dengue positive cases. The median and range of ages were 28 (1-91), 35 (5-87) and 36 years (15-77) for non-malarial, P. vivax and P. falciparum cases, respectively.

Using the Krustal-Wallis test to compare the four groups (see Table 1), median values of WBCs, PLTs, plateletcrit (PCT) and \%LYM were lower in malaria and dengue samples compared to negative ones with a statistical difference $(\mathrm{p}<0.05)$. Contrary, the median values of mean corpuscular hemoglobin concentration $(\mathrm{MCHC})$, mean platelet volume (MPV), platelet distribution width (PDW), and \%MON were higher than negative samples. RBC, HGB, hematocrit (HCT), mean corpuscular volume (MCV), mean corpuscular hemoglobin $(\mathrm{MCH})$ and granulocytes percentage (\%GRA) showed no significant difference in median between the four groups.

The medians of the parameters that had a significant difference between $P$. vivax infected and negative samples were WBCs, MCH, MCHC, RDW, PLTs, MPV, PCT, PDW and CRP. Similarly, P. falciparum cases had the same parameters with significant differences except in $\mathrm{MCH}, \mathrm{MCHC}$ and RDW.

Comparing both malaria infected cases with dengue, only PLT, PCT and CRP medians had a significant difference, which makes it difficult to differentiate between both diseases when observing solely the hematology parameters affection. The greatest difference of median between malaria and dengue infected patients was observed with CRP (Fig. 1).

\section{Parasitemia association with different hematology parameters}

To inspect the affection of the hematology parameters regarding the parasite density observed in peripheral blood, only the data-set of $P$. vivax samples in the current study had enough data variation to provide interesting results. The distribution of parasitemia in $P$. falciparum samples of the present study is limited, more than $60 \%$ of the cases had less than $0.2 \%$ of parasitemia, thus the analysis of associating parasitemia with the impact on hematology parameters was performed solely for the $P$. vivax set of data.

The parasitemia percentage establishes the relation of the number of parasites found in thin smears against the total count of RBCs and was calculated with the following formula:

$$
\text { \%Parasitemia }=\frac{\frac{\# \text { Parasites }}{100 W B C}\left(\frac{\# W B C}{\mu L}\right)}{\frac{\# R B C}{\mu L}} \times 100
$$

The total counts of WBCs and RBCs were retrieved from the hematology analyzer.

The parasitemia ranged from 0.00005 to $2.15 \%$ and no cases of high parasitemia ( $>5 \%$ ) were found. Table 2 describes the Spearman's rank correlation coefficients of the resulting parameters by the LC-667G in relation with the total parasitemia percentage of $P$. vivax positive samples. The parameters with statistically significant positive correlations with the total parasitemia percentage were WBC, RDW, PDW, \%LYM and CRP. Whereas negative correlations with statistical significance were observed in HGB, PLT, PCT and \%MON.

The CRP measurement value has many confounding factors including patient's age and sex. In order to evaluate the association and possible confounding factors of the available clinical variables with CRP value, a stratification of groups was created by matching patient age and sex between positive groups of P. falciparum $(\mathrm{n}=31$, mean age $=39.35, \mathrm{SD}=17.33)$ and $P$. vivax $(\mathrm{n}=31$, mean age $=39.54, \mathrm{SD}=17.66$ ). Table 3 shows the comparison of CRP medians between matched groups of $P$. vivax and $P$. falciparum, and each patient sex group. In addition, the Spearman's rank correlation coefficients between age and CRP value in each subgroup of malaria species were calculated. The null hypotheses of equality of medians were not rejected when comparing the CRP values between malaria species $(p$-value $=0.526)$, nor in patient sex of each malaria specie group. CRP correlates positively with 


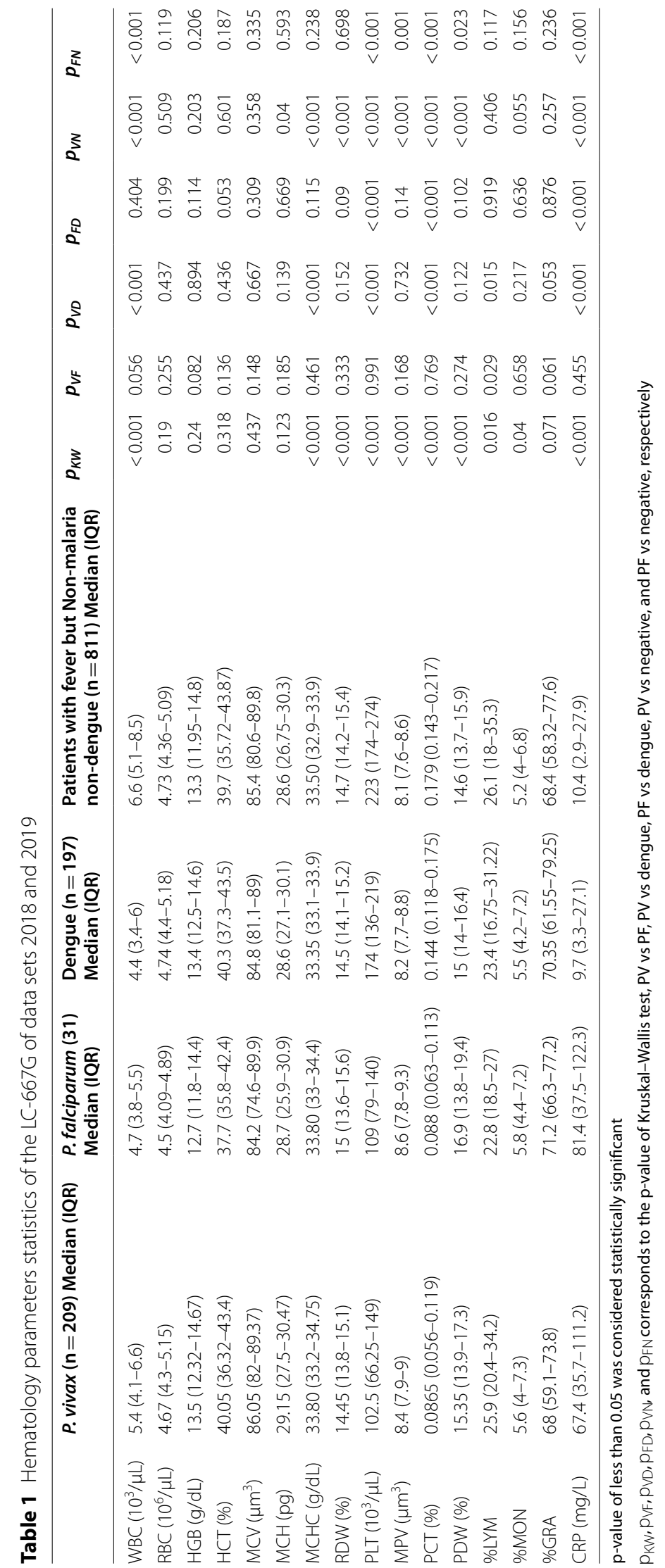



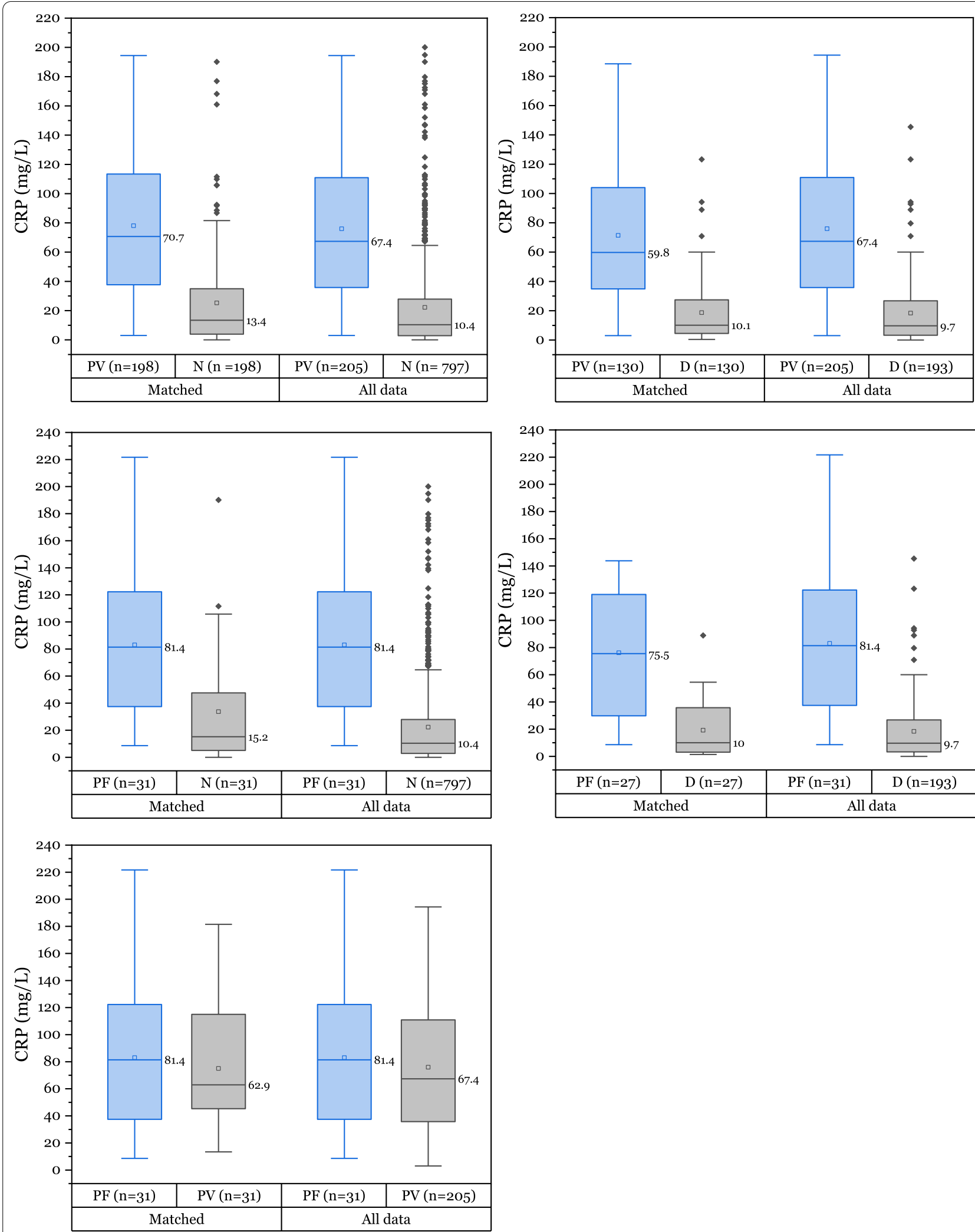

Fig. 1 Comparison of CRP distribution among different groups. P. vivax (PV), P. falciparum (PF), dengue (D) and non-malaria-dengue samples (N) distributions are represented with their corresponding median value and outliers. All comparisons left box-plots were matched in age ( \pm 2 years) and sex 
Table 2 Spearman's rank correlation coefficients and their corresponding $p$-value of the inspected parameters against the total parasitemia percentage of $P$. vivax samples

\begin{tabular}{lrr}
\hline & \%Parasitemia & p-value \\
& \multicolumn{1}{c}{$\mathbf{r}_{\mathbf{s}}$} & \\
\hline WBC & 0.285 & $<0.001$ \\
RBC & -0.128 & 0.068 \\
Hgb & -0.141 & 0.045 \\
Hct & -0.130 & 0.064 \\
MCV & -0.026 & 0.705 \\
MCH & -0.037 & 0.598 \\
MCHC & -0.037 & 0.594 \\
RDW & 0.138 & 0.049 \\
PLT & -0.303 & $<0.001$ \\
MPV & 0.078 & 0.265 \\
PCT & -0.283 & $<0.001$ \\
PDW & 0.179 & 0.010 \\
\%LYM & 0.143 & 0.042 \\
\%MON & -0.338 & $<0.001$ \\
\%GRA & -0.057 & 0.416 \\
CRP & 0.325 & $<0.001$ \\
\hline
\end{tabular}

10 samples from the original data set were incomplete on parasites counting therefore they were not contemplated for the calculation of Table 2 results

p-value of less than 0.05 was considered statistically significant

patient age in the $P$. falciparum group $(\mathrm{p}=0.008)$, but not in the $P$. vivax group $(\mathrm{p}=0.825)$.

\section{WBC histogram interference in the RBC ghosting area}

In the LC-667G CRP HORIBA analyzer, an abnormal peak located approximately at the $37 \mathrm{fL}$ channel in the WBC histogram appears mainly in malaria $P$. vivax samples with high density of large forms of parasites (see Fig. 2). The classification of parasite forms in the data set of 2018 was not available. Therefore only the parasite density information from dataset 2019 of $P$. vivax samples were used $(n=68,10$ samples were excluded due to incomplete information). A multiple linear regression model was calculated obtaining the following equation $y=0.184+2.238 \times 10^{-5} \alpha_{1}+6.007 \times 10^{-5} \alpha_{2}$, where $y$ represents the events per $\mu \mathrm{L}$ from 19.3fL to $43.9 \mathrm{fL}$ in the WBC histogram (refer Eq. 2), and $\alpha_{1}$ and $\alpha_{2}$ corresponds to the small and big parasite forms per $\mu \mathrm{L}$ identified in thin smears by microscopy, respectively. The contribution of large forms is approximately 2.7 times bigger than the contribution of small forms $\left(\alpha_{2} / \alpha_{1}\right)$ and the R-squared of the linear model is equal to 0.707 (see Fig. 3). Additionally, from the correlation matrix, the small and large forms of parasitemia showed a correlation of -0.315 .

$$
\begin{aligned}
& \text { Events per } \mu L \text { from } 19.3 \mathrm{f} \mathrm{L} \text { to } 43.9 \mathrm{fL} \\
& =\frac{\text { Sum events } 19.3 \mathrm{fL} \text { to } 43.9 \mathrm{fL}}{\text { Total events of } W B C \text { hist }} \\
& \quad \times\left(\# W B C \times 10^{3}\right)\left[\frac{\text { events }}{\mu L}\right]
\end{aligned}
$$

Large forms of parasites in P. falciparum samples are barely seen in peripheral blood due to sequestration in the spleen, thus interference in the $\mathrm{RBC}$ ghosting area of the WBC histogram will be smaller than in $P$. vivax samples where large forms of parasites remain in peripheral blood (compare subplots of Fig. 2). In the analyzed set of data, different interferences not-related to parasite inclusion located in the 37-fL area are displayed in Fig. 2. These interferences include platelet aggregations, macro platelets, and nRBCs.

\section{Analysis on the association and performance of hematology parameters and CRP to identify malaria}

From the statistically significant medians between malaria positive samples and the remaining samples (refer to Table 1), excluding derived parameters like PCT and PDW, calculations of the receiver operating characteristic (ROC) curve were included in the study to understand the clinical relevance of each abnormal parameter to predict malaria infection. Figure 4 shows the ROC curves that analyze matched malaria positive samples against negative and dengue samples. The AUC of the

\begin{tabular}{|c|c|c|c|c|c|}
\hline CRP (mg/L) & & & & & \\
\hline P. vivax (31) & & & P. falciparum (31) & & p-value \\
\hline $\mathrm{Md}=62.9$ & & & $\mathrm{Md}=81.4$ & & 0.526 \\
\hline Age correlation & & & Age correlation & & \\
\hline$r s=0.041, p=0.825$ & & & $r s=0.465, p=0.008$ & & \\
\hline Male (22) & Female (9) & $p$-value & Male (22) & Female (9) & p-value \\
\hline$M d=67.45$ & $M d=50.4$ & 0.727 & $\mathrm{Md}=75.15$ & $M d=88.6$ & 0.372 \\
\hline
\end{tabular}
inspected parameters is displayed in Table 4 along with

Table 3 CRP value association with malaria species, patient sex and age

A matching method of patient sex and age between positive $P$. vivax and $P$. falciparum groups was conducted

p-value of less than 0.05 was considered statistically significant 

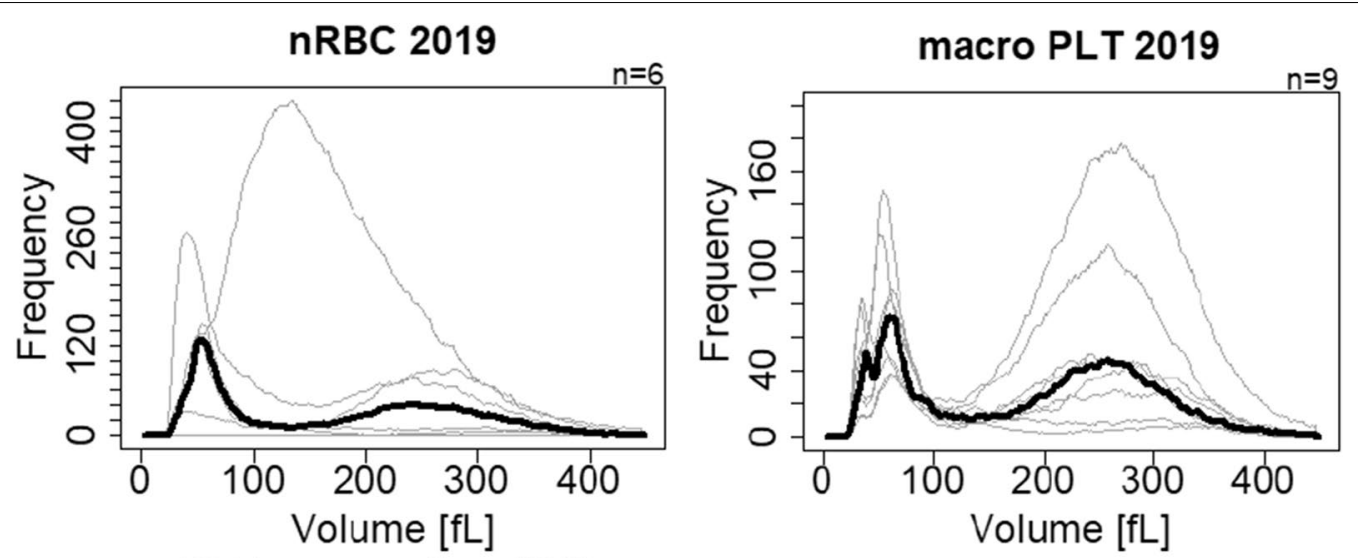

\section{PLT aggregation 2019}
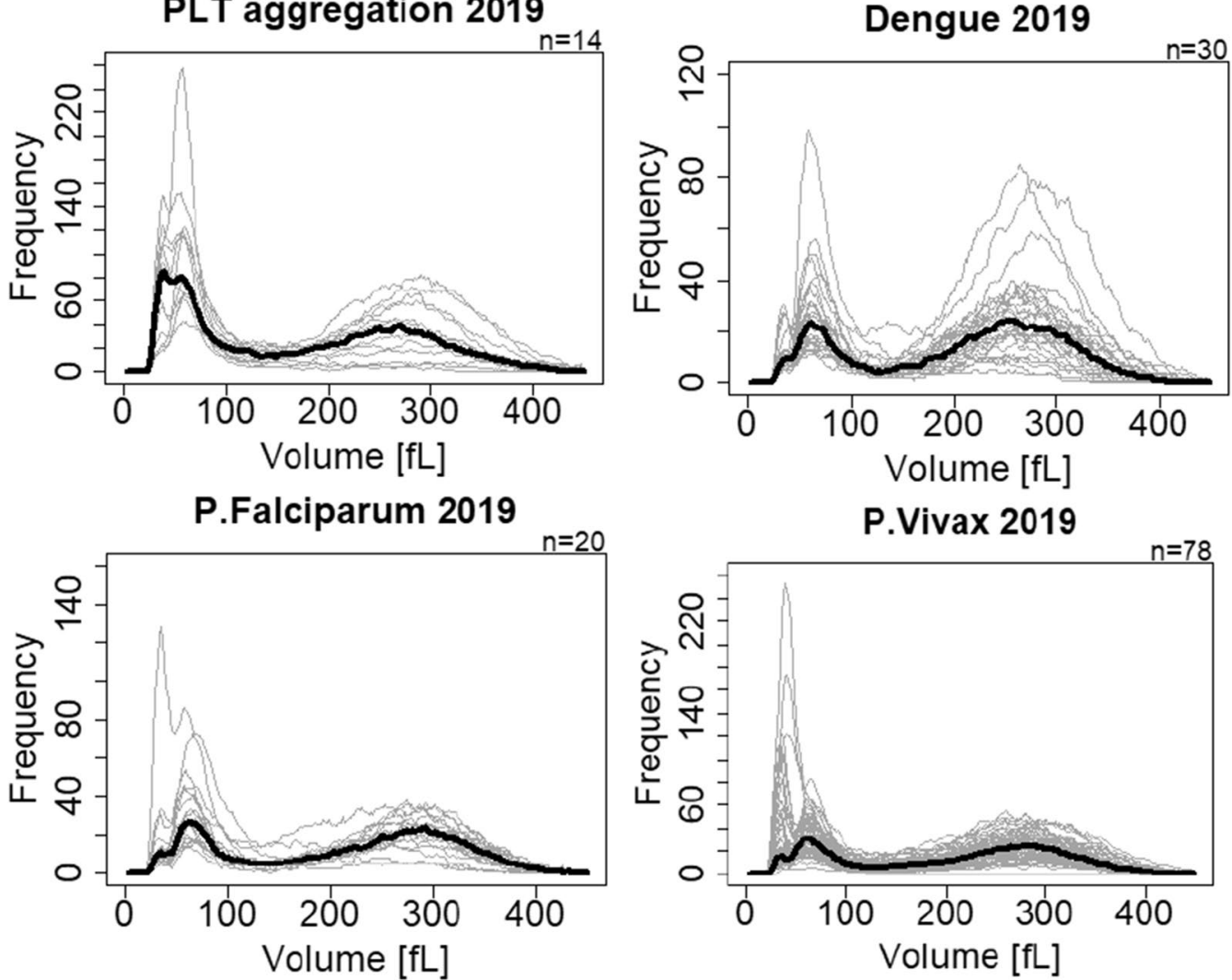

Fig. 2 WBC histograms of 2019 data-set with interferences around 37-fL. Bold lines represent the median of the histograms

the sensitivity, specificity, and odds ratio for the cut-off value using the Youden index.

\section{Discussion}

The most commonly reported affections on hematology parameters observed in malaria infection are thrombocytopenia, anemia, and leucopenia [16, 17]. Thrombocytopenia in malaria infections occurs due to complex platelet roles and abnormalities, including enabling cytoadhesion of iRBCs to endothelial cells, clumping and killing iRBCs [18], coagulation disturbances, bone marrow alterations, antibody-mediated platelet destruction, systemic platelet activation, vascular pooling, and oxidative stress [19]. Moreover, in hematological analyzers, agglutination of PLTs would lead to an apparent platelet loss due to shortcomings of the impedance method [20]. In the present study, thrombocytopenia $\left(<15010^{3} / \mu \mathrm{L}\right)$ was predominantly observed in malaria $P$. falciparum cases $(83.87 \%)$, rather than $P$. vivax $(76.44 \%)$, which is similar to the observations reported in $[21,22]$. Authors 


\section{Multiple linear regression model for PV parasitemia and WBC abnormal events}

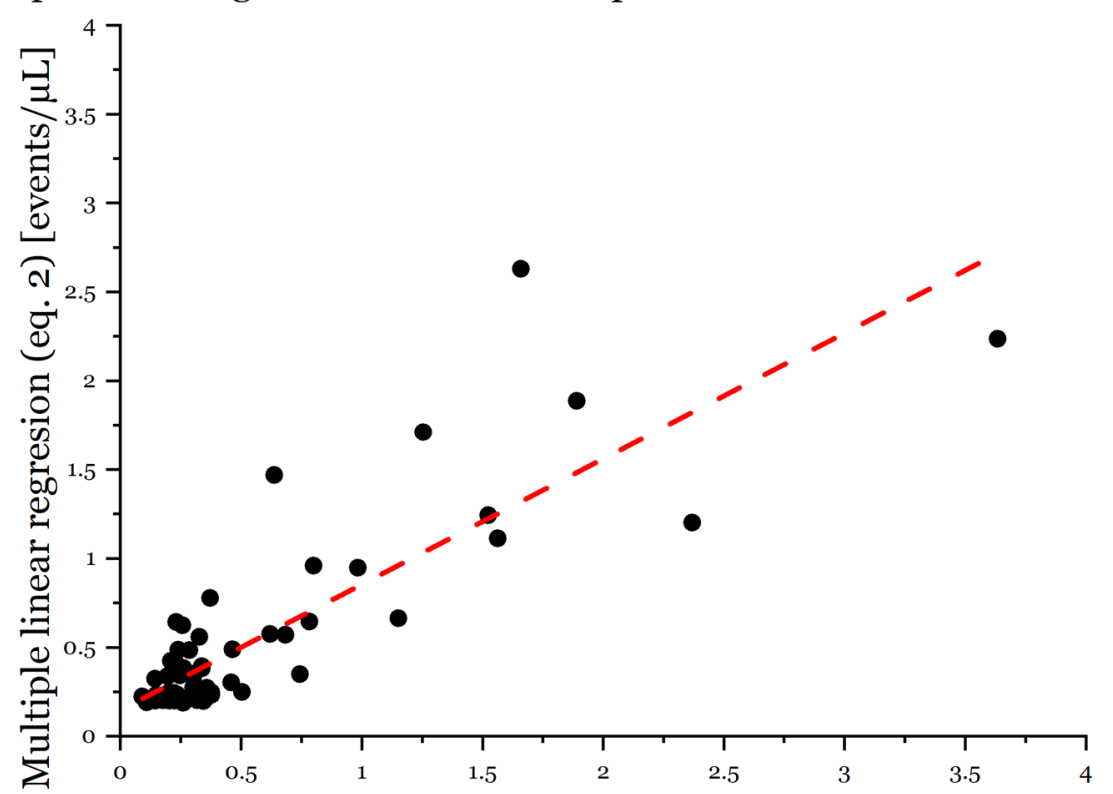

Events in the 19.3 - 43.9fL WBC histogram range [events/ $\mu \mathrm{L}$ ]

Fig. 3 Multiple linear regression model between the events before 43.9fL and parasite density. The vertical axis corresponds to the number of events from 19.3fL to 43.9fL in the WBC histogram and the horizontal axis to the parasite density of small and large forms of samples infected by malaria $P$. vivax. The regression model shows that the contribution of the linearity is mainly provided by large forms of $P$. vivax malaria infection

in [20] have concluded that in malaria cases, in addition to type of species, the severity of thrombocytopenia is also affected by parasite density. Furthermore, supporting this observation, our data indicates a negative correlation in P. vivax parasite density with PLT $(\mathrm{p}<0.001)$. To analyze the thrombocytopenia dependency on malaria species, the analysis must considers the parasite load and patient condition, as pointed out in a study where parasitemia was significantly higher in individuals with thrombocytopenia only in $P$. vivax, but not in P. falciparum infections [23].

Among the hematology parameters with significant statistical difference in medians between malaria positive and negative samples, platelets related parameters, especially MPV, should be further analyzed due to the strong affection with the elapsed time between collection and measurement of sample.

\section{Interference in the WBC channel and association with parasitemia}

In both three and five part differential ACCs a peak around the $\mathrm{RBC}$ ghosting area in the WBC histogram has been identified as one of the main abnormalities to predict malaria infection [24-29]. In the present study, the number of events within this abnormal peak were correlated with the parasite density of $P$. vivax large forms, supporting the same observation of moderate correlation $(r=0.707)$ in [28]. The abnormal peak under malaria infection is caused mainly by the lysing-resistant large forms of parasites, regardless of the species, and is probably affected by the presence of PLT aggregation and/or macro PLTs. The abnormal peak is less often observed in $P$. falciparum infections as the larger forms are sequestered by the spleen. Mature trophozoites and schizonts in $P$. falciparum infections are barely present in peripheral blood samples. According to [30], the presence of schizontaemia, especially in P. falciparum infections, can be used as a marker for disease severity. Therefore a semiquantitative estimation of schizontaemia can be inferred from the events located before the $37 \mathrm{fL}$ channel of the LC-667G WBC histogram and might help to assess the severity of malaria infections.

The abnormal peak in the WBC histogram is not exclusively due to malaria iRBCs. Common interferences include RBC lysed debris, PLT aggregations, macro-PLTs, nRBCs, and others. Researchers used this abnormal peak along with two other parameters of the LH780 (Beckman Coulter) to identify malaria infections having an $83.1 \%$ specificity but failed to discriminate from samples with nRBCs [28]. The distribution pattern of the abnormal peak might provide a way to differentiate the exact cause of it. It was observed that PLT aggregations or macro 

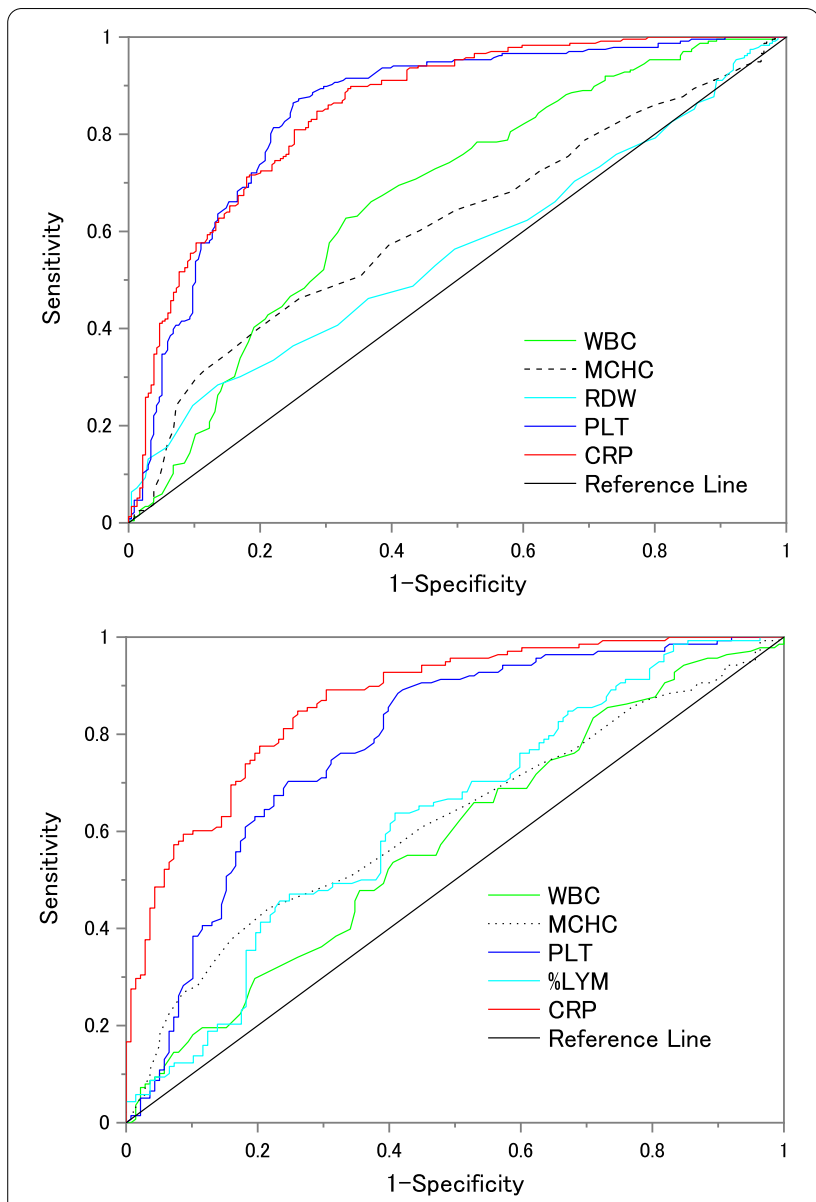

Fig. 4 Upper graph: ROC curve of all malaria positives against negative samples. Lower graph: ROC curve of all malaria positives against dengue samples. Compared groups were matched in age ( \pm 2 years) and sex, 236 and 138 matched samples for negative and dengue groups, respectively
PLTs show a bigger/wider distribution due to the random nature of formation, whereas gametocytes, schizonts and late stages of trophozoites show a narrower size distribution having a smaller dispersion close to the abnormal peak area.

Moreover, considering the parameters that are correlated with the total percentage of parasitemia such as WBC, PLT, and CRP, it might be possible to assess the level of parasitemia in combination with the WBC histogram analysis, especially in $P$. vivax infections. The positive and negative correlations of the $P$. vivax parasitemia with \%LYM $(\mathrm{p}=0.042)$ and \%MON $(\mathrm{p}<0.001)$ respectively, could be related to the interference in the WBC histogram, which suggests an incorrect counting of WBC subpopulations when iRBCs are present.

\section{A parameter to increase the specificity of detecting malaria: CRP}

In low cost ACCs where the Coulter principle is the only technology responsible for differentiating the WBC population into three parts based on volume, the identification of malaria or dengue infected samples is rather difficult due to the impossibility of differentiating granulocytes populations. Addition of CRP increases the specificity of detecting malaria due to the inflammatory response secondary to parasite infection.

Supporting observations of [31-35], in this study no significant difference of CRP value was observed between medians of matched $P$. vivax and $P$. falciparum groups $(\mathrm{p}=0.526)$. The non-statistical significance in age and CRP correlation in the P. vivax group might be affected by the wider distribution of parasite density (cf. P. falciparum group). The parasitemia was not

Table 4 AUC, Youden's index and accuracy results of single parameters with statistical significance between medians of malaria positive, malaria negative, and dengue positive samples

\begin{tabular}{|c|c|c|c|c|c|c|c|c|}
\hline \multirow[t]{2}{*}{ Parameter } & \multicolumn{4}{|c|}{ Malaria vs Negative samples } & \multicolumn{4}{|c|}{ Malaria vs dengue samples } \\
\hline & AUC & Cut-off value at YI & $\begin{array}{l}\text { Sens.\% } \\
\text { Spe. \% }\end{array}$ & Odds ratio (Cl 95\%) & AUC & Cut-off value at YI & $\begin{array}{l}\text { Sens.\% } \\
\text { Spe. \% }\end{array}$ & Odds ratio ( $\mathrm{Cl} 95 \%)$ \\
\hline WBC & 0.672 & $\begin{array}{l}\leq 5.75 \\
\left(10^{3} / \mu \mathrm{L}\right)\end{array}$ & $\begin{array}{l}62.71 \% \\
66.94 \%\end{array}$ & $3.40(2.33-4.97)$ & 0.580 & $\begin{array}{l}\geq 4.35 \\
\left(10^{3} / \mu \mathrm{L}\right)\end{array}$ & $\begin{array}{l}65.94 \% \\
47.10 \%\end{array}$ & $1.72(1.06-2.80)$ \\
\hline $\mathrm{MCHC}$ & 0.613 & $\begin{array}{l}\geq 33.95 \\
(\mathrm{~g} / \mathrm{dL})\end{array}$ & $\begin{array}{l}39.83 \% \\
80.42 \%\end{array}$ & $2.71(1.79-4.11)$ & 0.620 & $\begin{array}{l}\geq 33.85 \\
(\mathrm{~g} / \mathrm{dL})\end{array}$ & $\begin{array}{l}44.20 \% \\
78.26 \%\end{array}$ & $2.85(1.68-4.82)$ \\
\hline RDW & 0.558 & $\begin{array}{l}\leq 13.85 \\
(\%)\end{array}$ & $\begin{array}{l}28.39 \% \\
86.44 \%\end{array}$ & $2.52(1.58-4.03)$ & & & & \\
\hline PLT & 0.849 & $\begin{array}{l}\leq 161.5 \\
\left(10^{3} / \mu \mathrm{L}\right)\end{array}$ & $\begin{array}{l}86.44 \% \\
75.00 \%\end{array}$ & $19.12(11.89-30.75)$ & 0.780 & $\begin{array}{l}\leq 160.5 \\
\left(10^{3} / \mu \mathrm{L}\right)\end{array}$ & $\begin{array}{l}88.40 \% \\
58.69 \%\end{array}$ & $10.83(5.81-20.17)$ \\
\hline$\%$ LYM & & & & & 0.628 & $\begin{array}{l}\geq 24.6 \\
(\%)\end{array}$ & $\begin{array}{l}63.76 \% \\
59.12 \%\end{array}$ & $2.54(1.56-4.14)$ \\
\hline CRP & 0.852 & $\begin{array}{l}\geq 27.65 \\
(\mathrm{mg} / \mathrm{L})\end{array}$ & $\begin{array}{l}84.74 \% \\
71.36 \%\end{array}$ & $13.84(8.79-21.80)$ & 0.867 & $\begin{array}{l}\geq 26.85 \\
(\mathrm{mg} / \mathrm{L})\end{array}$ & $\begin{array}{l}84.78 \% \\
73.91 \%\end{array}$ & $15.78(8.66-28.76)$ \\
\hline
\end{tabular}

The sensitivity, specificity, and odds ratios were calculated with the cut-off values of the Youden's index. Negative samples were matched with malaria positive samples in age and sex 
included in the current study's matching method due to the limited variation on $\mathrm{P}$. falciparum positive samples. However, for future analyses on CRP, parasitemia should be also considered and treated as a confounding variable.

Dengue and malaria in many countries are coendemic diseases that present similar clinical presentations. The importance of distinguishing them has been reported by [36], who developed a tree decision model for discriminating malaria and dengue infections. Dengue samples collected in the present study had a lower count of WBCs and MCHC in comparison to both malaria species supporting observations of [36]. Only for $P$. vivax samples, the difference between medians was statistically significant. Nonetheless, both variables have a poor clinical relevance for differentiating malaria from dengue samples using the Youden's index (AUC of $\mathrm{WBC}=0.580$ and AUC of MCHC 0.620). In contrast, PLT medians of both species of malaria infections were lower than dengue cases $(\mathrm{p}<0.001)$ and the odds ratio of malaria positive patients having less than $160 \times 10^{3}$ platelets $/ \mu \mathrm{L}$ was 10.83 . Discrepancy of results with [36] might be related to the severity of each disease. For example, the hematology parameters affection in dengue infections is greatly dependent on the day of fever. Unfortunately, current data does not include any information about the fever progression of dengue infected individuals.

The CRP parameter showed the most significant difference between malaria and dengue samples. From our current data set, patients with malaria are 15.78 times more likely to present CRP greater than $26.85 \mathrm{mg} / \mathrm{L}$, compared to dengue patients. In comparison with negative patients with fever, patients with malaria have a smaller odds ratio (OR 13.84, CI 8.70-21.80) to present CRP greater than $27.65 \mathrm{mg} / \mathrm{L}$. Although CRP is not a specific biomarker for malaria, the response of CRP to virus infections is rather small, making it a good parameter to understand the etiology of the infection and to help differentiate malaria and dengue from similar hematology parameters. Nevertheless, dengue and other non-viral infections can co-exist in a single sample, thus relying only on CRP should not be an exclusive criteria in differentiating both diseases.

In general, hematology parameters and CRP depend on clinical characteristics and might vary upon demographics. Additionally, the immune response against malaria, dengue or other infections is related to the condition of the patient and the disease's stage. Caution to generalize the current results must be taken considering the limited distribution of parasites in the inspected samples (particularly in P. falciparum), the single location of the study, and the bias selection from retrospective results.

\section{Conclusions}

The LC-667G CRP, a three-part differential hematology analyzer has the potential to not only trigger malaria diagnosis confirmation, but also to assess the severity of the infection due to simultaneous CRP estimations. The current study suggests that CRP could be helpful for distinguishing malaria from dengue. In addition to CRP, the abnormal peak located around the $37 \mathrm{fL}$ channel in the WBC histogram can provide a good estimation of the number of large parasites present during the infection. However the calculation might be interfered by other particles such as nRBCs and PLT aggregations. The combination of providing hematology parameters plus CRP values in a hematology analyzer makes a preferred method for screening malaria on a routine basis in cases of any infection suspicion. However, the sole inspection of these abnormal parameters should not by any means replace the use of the golden standard methods for diagnosing malaria (i.e., microscopy inspection and RDTs).

Our current data set is limited by the number of $P$. falciparum samples. The discussion and observations provided were focused on $P$. vivax positive cases. Further research following this study should be oriented to measure $P$. falciparum samples, to evaluate the potential of using hematology analyzers to provide additional information to the RDTs, and to monitor anti-malaria drugs treatments.

\section{Abbreviations \\ WHO: World Health Organization; RDT: Rapid diagnostic test; ACC: Automated cell counter; CBC: Complete blood count; WBC: White blood cell; LYM: Lym- phocytes; MON: Monocytes; RBC: Red blood cell; iRBC: Infected red blood cell; HGB: Hemoglobin; TLC: Total lymphocyte count; RDW: Red cell distribution width; CRP: C-reactive protein; POCT: Point of care testing; PLT: Platelet; PCT: Plateletcrit; MCHC: Mean corpuscular hemoglobin concentration; MPV: Mean platelet volume; PDW: Platelet distribution width; HCT: Hematocrit; MCV: Mean corpuscular volume; MCH: Mean corpuscular hemoglobin; GRA: Granulocytes; CV: Coefficient of variation; nRBC: Nucleic red blood cell.}

\section{Supplementary Information}

The online version contains supplementary material available at https://doi. org/10.1186/s12879-021-06704-5.

Additional file 1: Annex 1. Annex 1 QUADAS-2 analysis.

Additional file 2: Annex 2. Raw data.

\section{Acknowledgements}

We thank the laboratory team of Dr. Dharap Diagnostic Centre for their support, Masato Nojiri for his statistical review, and Akika Ohashi and Masashi Nishimori for their support in the statistical and data analysis.

\section{Authors' contributions}

PD, SR, and JN designed the study. JN drafted the manuscript and performed the data analysis. PD collected all samples, performed the smear inspections and clinical observations. SR and PD reviewed and corrected the manuscript. All authors read and approved the final manuscript. 


\section{Funding}

The study was funded by HORIBA Ltd., and helped with the analysis and interpretation of the hematology analyzer results.

\section{Availability of data and materials}

The datasets used and/or analyzed during the current study are available in the Additional file 2: "Annex 2 Raw data". The age and gender information was removed to protect patient anonymity.

\section{Declarations}

\section{Ethics approval and consent to participate}

For the current study, the historical documented data was further anonymized in coded fashion in order to protect patient confidentiality in accordance with local ethical or IRB guidelines, according to national and international standards for the conduct of clinical studies including 21 CFR Parts 50 and 56 and International Conference on Harmonization (ICH) E6-Good Clinical Practice Consolidated Guideline. Ethical clearance was not obtained as the study was carried out as a retrospective review of the available raw instrument and historical patient data, without any collection of extra specimen or monetary charges to patients and did not involve any communication of results of the study to the clinician, so as to affect the diagnostic and therapeutic management of the patients.

\section{Consent for publication}

Not applicable.

\section{Competing interests}

JN and SR are employees of HORIBA Ltd. PD is an external consultant of HORIBA Ltd.

\section{Author details}

${ }^{1}$ Horiba Medical, Kyoto, Japan. ${ }^{2}$ Dharap's Diagnostic Center, Mumbai, India.

${ }^{3}$ Horiba Medical, Montpellier, France.

Received: 6 September 2020 Accepted: 16 September 2021

Published online: 26 September 2021

\section{References}

1. WHO. World malaria report 2018. Geneva: World Health Organization; 2018. http://www.who.int/malaria/publications/world-malaria-report2018/en/. Accessed 20 Jan 2020.

2. McMorrow ML, Aidoo M, Kachur SP. Malaria rapid diagnostic tests in elimination settings - can they find the last parasite? Clin Microbiol Inf. 2011;17:1624-31.

3. Campuzano-Zuluaga G, Hänscheid T, Grobusch MP. Automated haematology analysis to diagnose malaria. Malar J. 2010;9:346.

4. Mendelow BV, Lyons C, Nhlangothi P, Tana M, Munster M, Wypkema E, Liebowitz L, Marshall L, Scott S, Coetzer TL. Automated malaria detection by depolarization of laser light. Br J Haematol. 1999;104(3):499-503.

5. Jain M, Gupta S, Jain J, Grover RK. Usefulness of automated cell counter in detection of malaria in a cancer set up-Our experience. Indian J Pathol Microbiol. 2012;55:467-73.

6. Huh J, Jung J, Yoon H, Chung W. Pseudoeosinophilia associated with malaria infection determined in the Sysmex XE-2100 hematology analyzer. Ann Hematol. 2005;84:400-2.

7. Fourcade C, Casbas MJ, Belaouni H, Gonzalez JJ, Garcia PJ, Pepio MA Automated detection of malaria by means of the haematology analyser CoulterR GEN.STM. Clin Lab Haematol. 2004:26:367-72.

8. Sun Y, Xiang D, Chen C, et al. Infected RBC flag/parameter provided by Mindray BC-6800 haematology analyzer aid the diagnosis of malaria. Malar J. 2019;18:262.

9. Jairajpuri ZS, Rana S, Hassan MJ, Nabi F, Jetley S. An analysis of hematological parameters as a diagnostic test for malaria in patients with acute febrile illness: an institutional experience. Oman Med J. 2014;29(1):12-7.

10. Mu AK, Bee PC, Lau YL, Chen Y. Identification of protein markers in patients infected with Plasmodium knowlesi, Plasmodium falciparum and Plasmodium vivax. Int J Mol Sci. 2014;15(11):19952-61.
11. Paul R, Sinha PK, Bhattacharya R, Banerjee AK, Raychaudhuri P, Mondal J. Study of $C$ reactive protein as a prognostic marker in malaria from Eastern India. Adv Biomed Res. 2012;1:41.

12. Sarfo BO, Hahn A, Schwarz NG, et al. The usefulness of C-reactive protein in predicting malaria parasitemia in a sub-Saharan African region. PLOS ONE. 2018;13:e0201693.

13. McGuire W, D'Alessandro U, Olaleye BO, Thomson MC, Langerock $P$, Greenwood BM, et al. C-reactive protein and haptoglobin in the evaluation of a community-based malaria control programme. Trans R Soc Trop Med Hyg. 1996;90(1):10-4.

14. Andrade BB, Reis-Filho A, Souza-Neto SM, et al. Severe Plasmodium vivax malaria exhibits marked inflammatory imbalance. Malar J. 2010:9:13.

15. Epelboin L, Boullé C, Ouar-Epelboin S, Hanf M, Dussart P, Djossou F, Nacher M, Carme B. Discriminating malaria from dengue fever in endemic areas: clinical and biological criteria, prognostic score and utility of the C-reactive protein: a retrospective matched-pair study in French Guiana. PLoS Neglect Trop Dis. 2013;7:1.

16. Awoke N, Arota A. Profiles of hematological parameters in Plasmodium falciparum and Plasmodium vivax malaria patients attending Tercha General Hospital, Dawuro Zone, South Ethiopia. Infect Drug Resist. 2019;12:521-7.

17. Ullah I, Ali MU, Ali S, Ahmad R, et al. Hematological profile of patients having malaria-positive peripheral blood smears: a cross-sectional study at a diagnostic research center in Khyber Pakhtunkhwa, Pakistan. Cureus. 2019;10(9):1.

18. McMorran BJ, Marshall VM, de Graaf C, et al. Platelets kill intraerythrocytic malarial parasites and mediate survival to infection. Science. 2009;323(5915):797-800.

19. Lacerda MV, Mourao MP, Coelho HC, Santos JB. Thrombocytopenia in malaria: who cares? Mem Inst Oswaldo Cruz. 2011;106:1.

20. Kho S, Barber BE, Johar E, et al. Platelets kill circulating parasites of all major Plasmodium species in human malaria. Blood. 2018;208:1332-44.

21. Manmeet KG, Manisha M, Sachan B, et al. Thrombocytopenia in malaria and its correlation with different types of malaria. Ann Trop Med Public Health. 2013;6:197-200.

22. Kotepui M, Phunphuech B, Phiwklam N, et al. Effect of malarial infection on haematological parameters in population near Thailand-Myanmar border. Malar J. 2014;13:218.

23. Da Costa Lima-Junior J, Nunes Rodrigues-da-Silva R, Araujo Pereira V, et al. Cells and mediators of inflammation (C-reactive protein, nitric oxide, platelets and neutrophils) in the acute and convalescent phases of uncomplicated Plasmodium vivax and Plasmodium falciparum infection. Mem Inst Oswaldo Cruz. 2012;107:8.

24. Akinosoglou KS, Solomou EE, Gogos CA. Malaria: a haematological disease. Hematology. 2012;17(2):106-14.

25. Kotepui M, Piwkham D, PhunPhuech B, Phiwklam N, Chupeerach C, Duangmano S. Effects of malaria parasite density on blood cell parameters. PLOS ONE. 2020;10:e0121057.

26. Briggs C, Costa A, Freeman L, Aucamp I, Ngubeni B, Machin S. Development of an automated malaria discriminant factor using VCS technology. Am J Clin Pathol. 2006;126:691-8.

27. Singh A, Narang V, Sood N, Garg B, Gupta VK. Malaria diagnosis using automated analysers: a boon for hematopathologists in endemic areas. J Clin Diagn Res. 2015;9(10):EC05-8.

28. Yoon J, Yun SG, Kim J, Jung YN, Koh YE, Nam JH, et al. Evaluation of the LH780 hematology analyzer for detection and therapeutic monitoring of malaria: cross-reactivity with nucleated RBCs. Acta Trop. 2016;164:425-30.

29. Sharma S, Sethi N, Pujani M, Kushwaha S, Sehgal S. Abnormal WBC scattergram: a clue to the diagnosis of malaria. Hematology. 2013;18(2):101-5.

30. van Wolfswinkel ME, de Mendonça Melo M, Vliegenthart-Jongbloed K, et al. The prognostic value of schizontaemia in imported Plasmodium falciparum malaria. Malar J. 2012;11:301.

31. Hien S, Yeboah OR, Adou H, N'Guessan K, Kouacou APV, Dassé SR. Study about relationship between $C$-reactive protein (CRP) and other indicators in children with malaria. J Infect Dis Immunity. 2016;8(2):10-7. 
32. Pelkonen T, Albino A, Roine I, Bernardino L, Peltola H. C-reactive protein in children with malaria in Luanda, Angola: a prospective study. Trans R Soc Trop Med Hyg. 2015;109(8):535-7.

33. Utuk EE, Ikpeme EE, Udo JJ, Akpan MU. Predictors of C-reactive protein response in children infected with Plasmodium falciparum malaria. East Afr Med J. 2014;91(1):1-7.

34. Hurt N, Smith T, Teuscher T, Tanner M. Do high levels of C-reactive protein in Tanzanian children indicate malaria morbidity. Clin Diagn Lab Immunol. 1994;1 (4):437-44.

35. Gillespie SH, Dow C, Raynes JG, Behrens RH, Chiodini PL, McAdam KP. Measurement of acute phase proteins for assessing severity of Plasmodium falciparum malaria. J Clin Pathol. 1991;44(3):228-31.

36. Kotepui M, PhunPhuech B, Phiwklam N, Uthaisar K. Differentiating between dengue fever and malaria using hematological parameters in endemic areas of Thailand. Infect Dis Poverty. 2017;6:1-9.

\section{Publisher's Note}

Springer Nature remains neutral with regard to jurisdictional claims in published maps and institutional affiliations.
Ready to submit your research? Choose BMC and benefit from:

- fast, convenient online submission

- thorough peer review by experienced researchers in your field

- rapid publication on acceptance

- support for research data, including large and complex data types

- gold Open Access which fosters wider collaboration and increased citations

- maximum visibility for your research: over $100 \mathrm{M}$ website views per year

At BMC, research is always in progress.

Learn more biomedcentral.com/submissions 